ing the cultures with radium.

We wish to express our thanks to Mr. Berlinicke, of the firm of Hugo Lieber \& Co., who was kind enough to loan us the radium used in these experiments, and to Mr. Bagg, who assisted us in our observations.

JACQUES LOEB

F. W. BANCROFT

ROCKEFELLER INSTITUTE FOR

Medical REsearch

\section{AN EXPERIMENT IN DOUBLE MATING}

IN my "Inheritance in Silkworms, I.," $(1908)^{1}$ I called attention (pp. 37-39) to the beginnings of an experiment in double mating. Only the $F_{1}$ generations following a few matings had been obtained at that time, but they gave such promise of interest that I determined to continue the experiment and to widen it. I have now in hand the notes on 85 silkworm broods belonging to this double mating experiment series of 1910. Some of these broods are the $\mathrm{F}_{3}$ generation from the original 1907 double matings, while others are $F_{2}$ or $F_{1}$ generations from the original 1908 or 1909 double matings. Taken together the notes of these various 1907-10 rearings from double matings are sufficient to pose some suggestive queries.

By the double mating of silkworms I mean the mating of a female of one race with two males representing different races, one of them usually of the same race of the female, the other of another race. Races are chosen which are readily distinguished by a difference in cocoon color, as yellow or white, or in larval pattern, as banded and unbanded. The silkworm is polygamous and polyandrous, both males and females usually mating more than once before egg-laying begins. Or this repeated mating may continue after egg-laying has begun.

Moths to be experimentally double-mated are reared from carefully isolated cocoons, and

1" Inheritance in Silkworms, I.," Leland Stanford Junior University Publications, University Series, No. 1, 89 pp., 4 plates, 2 text figs., 1908. Address Librarian, Stanford University, California. the two matings are made to take place immediately following one another for equal or definitely determined unequal periods of coupling, and always before any egg-laying by the female. The young produced from the eggs of each double-mated female are reared isolated in separate trays, which are covered over during the later larval life (possible straggling time).

In any consideration of the results of such repeated mating the unusual way in which the eggs of insects (at least of the silkworm moth and hosts of others) are fertilized must be remembered. This way is, simply, that the male fertilizing cells, the spermatozoa, are received by the female at mating into a special sac or receptacle, the spermatheca (there may be several spermathecæ, as in flies) in which the spermatozoa remain alive and active. This spermatheca, a diverticulum of the oviduct, is situated near its external opening, the vagina. As the unfertilized eggs of the moth pass slowly down from the ovarial tubes into the oviduct they lack only fertilization to be entirely ready for development. They have already their full supply of yolk, they are already enclosed in their protecting envelopes (vitelline membrane and outer, firmer chorion). But these envelopes do not completely enclose the egg-mass; there is, at one pole of the egg, one or more small openings, the micropyle, through which the spermatozoa, issuing from the duct of the spermatheca as the eggs pass, enter the eggs. As soon as a single spermatozoan has entered, a jelly-like substance closes the micropyle and prevents polyfertilization.

Thus when the silkworm moth first mates she receives in her spermatheca, and holds there, a considerable number of spermatozoa representing the heritable characters of the male involved. When she couples again she receives another lot of spermatozoa, and if the second coupling is with a male of different race from the first these spermatozoa represent a new set of characters. What is going to be the result of this double mating as exhibited in the offspring?

It seems, at first thought, that this result 
should be nothing new; nothing surprising. We know already what to expect in any simple mating of different races of silkworms. As regards larval pattern and cocoon color the inheritance behavior is usually Mendelian. "Moricaud" (all dark) larval pattern is dominant over " tiger," or banded, pattern; banded pattern is dominant over unbanded (all light). Yellow cocoon color is dominant over white. And the relation of dominant and recessive is of the usual Mendelian character in $\mathrm{F}_{1}, \mathrm{~F}_{2}, \mathrm{~F}_{8}$ and succeeding generations. Now although there are two kinds (two races representing these alternative larval and pupal characters) of spermatozoa in the spermatheca of doublemated females, presumably but one spermatozoid finds its way into the egg, and fuses its nuclear matter with the egg nucleus. That is, the female, although double-mated, is presumably only single-fertilized.

As a matter of fact the inheritance behavior of the $F_{1}$ and succeeding generations derived from these double-mated females does not seem to bear out the simple presumption just stated. The presence in the body (spermatheca) of the female of two kinds of spermatozoa seems to disturb matters. The comfortable simplicity and regularity of Mendelian inheritance fails to maintain itself. The troubles of irregularity which have not been wholly wanting even in single mating silkworm experiments-and which I have termed in my account of several years' experience of these matings, "strain and individual idiosyncrasies," a term not looked on with favor by thorough-going Mendelians-these irregularities are accented in the double-mating experiments. The irregularities indeed almost assume a seeming of regularity; a nonMendelian regularity, if such a heresy is admissible.

I shall not try to give here the full data of my 85 "double-mating" lots of 1910 . But I shall give a considerable number of examples and a general statement of these results. Later, if worth while, all the data can be given; together, I may add, with the results of three or four years' more work in singlemating crossings to test further the inherit- ance of certain egg, larval and cocoon characters, already pretty fairly determined by the original series of several years whose results have been published.

I shall limit the examples referred to in this paper to matings among three races and shall refer only to cocoon characters. The three races are Istrian Yellow, a strong Austrian race producing large golden yellow cocoons; French Yellow, a race producing smaller, salmon yellow cocoons; and Bagdad White, a Turkish race producing large pure white cocoons. Bagdad White is a race whose white cocoon color, instead of being regularly recessive to yellow in crossings with yellow cocooning races, is sometimes dominant, as I have shown in my 1908 report (pp. 24-25, section on "strain and individual idiosyncrasies"). All these races have been bred as pure races by me for the last ten years; that is, races faithfully transmitting certain larval and cocoon characters.

In 1908 a Bagdad White female was mated with a French Yellow male from $9: 55$ A.M. to 11:55 A.M. and with a Bagdad White male from 11:55 A.M. to 1:55 P.M. The young, reared in 1908, were all white cocooners. One mating (1908) (single) among these young produced (1909) 111 white cocoons and 44 yellow cocoons; another produced all white cocoons; also another produced all white cocoons. A mating (1909) between two white cocooners out of the 111 white and 44 yellow lot, produced (1910) all white cocoons. A mating of two yellow cocooners produced (1910) 8 white cocoons and 40 yellow cocoons; another produced 9 white cocoons and 29 yellow cocoons. A mating (1908) between two white cocooners of one of the all white cocoon lots produced (1909) all white cocoons; and so did another. A mating (1909) of two white cocooners of one of these all white cocoon lots produced (1910) 15 white cocoons and 2 yellow cocoons (sick lot); another produced all white cocoons. A mating (1909) of two white cocooners from the other all white cocoon lot produced (1910) 17 white cocoons and 4 yellow cocoons; and another produced 14 white cocoons (both small sick lots). 
In 1907 a Bagdad White female was mated with a Bagdad White male from $9: 30$ A.M. to $10: 55$ A.M. and then with a French Yellow male from $10: 55$ A.M. to $12: 15$ P.M. From this mating there were produced (1908) 25 white cocoons and 13 yellow cocoons. Mating (1908) two of these white cocooners together produced (1909) a small lot divided equally between white cocoons and yellow cocoons (sick lot). Mating (1909) two of these white cocooners together produced (1910) a small lot of white cocooners containing one yellow cocoon (straggler?). (Wherever my records show a single yellow in an otherwise white lot or a single white in an otherwise yellow lot I prefer "straggling" to any other explanation!) A mating (1909) of two yellow cocooners produced (1910) a small yellow lot containing one white cocoon. A mating (1908) of a white and a yellow from the 25 white, 13 yellow lot produced (1909) a small lot composed equally of white and of yellow cocooners. Mating (1909) two of these white cocooners together produced (1910) 23 white cocoons and 2 yellow cocoons.

In 1907 another Bagdad White female was mated with a Bagdad White male from $9: 30$ A.M. to $10: 55$ A.M. and then with a French Yellow male from 10:55 A.M. to $12: 15$ P.M. From this mating there were produced (1908) 33 white cocoons and 19 yellow cocoons. Mating (1908) two of the yellows produced (1909) a small lot equally divided between yellow and white cocoons. Mating (1909) two of these white cocooners produced (1910) 9 white cocoons and one yellow. Mating (1908) a yellow and a white from the half yellow, half white $\mathrm{F}_{1}$ generation produced (1909) 22 white cocoons. And mating (1909) two of these together produced (1910) a small lot of all white cocooners. Mating (1908) another yellow with another white from the $\mathrm{F}_{1}$ generation produced (1909) 6 white cocoons and 10 yellow cocoons. Mating (1909) two of these yellow cocooners produced (1910) a small lot equally divided between yellow and white cocoons. Mating (1908) two white from the $F_{1}$ generation produced (1909) a small lot all white except for a single yellow cocoon. And mating (1909) two of these whites together produced (1910) an all white lot of cocoons.

In 1907 a French yellow female was mated with a French yellow male from $9: 15$ A.M. to $10: 45$ P.M. and then with a Bagdad white male from $10: 45$ A.M. to $12: 15$ P.M. From this mating there were produced 57 white cocoons and 74 yellow cocoons. Mating (1908) two yellows of this $\mathrm{F}_{1}$ generation produced (1909) 22 yellow and 8 white cocoons. Another similar mating (1908) produced (1909) 23 yellow and 2 white cocoons. And still another produced 14 yellow and 5 white cocoons. Mating (1908) a yellow and a white of the $\mathrm{F}_{1}$ generations produced (1909) 17 yellow and 19 white cocoons. Another similar mating (1908) produced (1909) 21 yellow and 17 white cocoons. Mating (1909) two whites of the $\mathrm{F}_{2}$ generation produced by two yellow parents produced (1910) an all white lot. Mating (1909) two more whites of this $\mathrm{F}_{2}$ lot produced (1910) another all white $F_{3}$ lot. Mating (1909) two yellows from this same $\mathrm{F}_{2}$ lot produced (1910) an all yellow lot. Mating (1909) another pair of these $\mathrm{F}_{2}$ yellows produced (1910) 20 yellow and 9 white cocoons. Mating (1909) two whites of the $F_{2}$ generation produced by a white $X$ yellow produced (1910) 25 whites, 11 yellows and a double cocoon spun together by a white cocooning larva and a yellow cocooning larva. Another mating (1909) of two whites from this same $F_{2}$ lot produced (1910) 19 white cocoons and 6 yellow cocoons.

In 1907 a French Yellow female was mated with a Bagdad White male from 9:40 A.M to 11:10 A.M. and then with a French Yellow male from 11:10 A.M. on to the death of the moths. This mating produced (1908) 14 white cocoons and 140 yellow cocoons. Mating (1908) two of the white cocooners produced (1909) 30 white cocoons and 10 yellow cocoons. Mating (1909) two of these $\mathrm{F}_{2}$ generation white cocooners produced (1910) 62 white cocoons and no yellows. Mating (1908) two more of the $F_{1}$ generation white cocooners produced (1909) 20 white cocoons and 10 yellow cocoons. Mating (1909) two of the $\mathrm{F}_{2}$ 
generation white cocooners produced (1910) a small sick lot, partly white and partly yellow. Mating (1909) two of the $\mathrm{F}_{2}$ generation yellow cocooners produced (1910) a small weak lot of 12 yellow cocoons and 2 white cocoons. Mating (1908) two of the $F_{1}$ generation yellow cocooners produced (1909) 18 yellow cocoons and 2 white cocoons. Mating (1909) two of the $F_{2}$ yellow cocooners produced 4 yellow cocoons and one white cocoon. Mating (1908) two more of the $F_{1}$ generation yellow cocooners produced (1909) 59 yellow cocoons and no white cocoons. Mating (1909) two of these $\mathrm{F}_{2}$ yellow cocooners produced (1910) 47 yellow cocoons and no white cocoons. Mating (1908) a yellow cocooner and a white cocooner of the same $F_{1}$ generation lot produced (1909) 18 white cocoons and 14 yellow cocoons. Mating (1909) two of these $\mathrm{F}_{2}$ white cocooners together produced (1910) 29 white cocoons and 11 yellow cocoons. Mating (1909) two of the yellow cocooners of the $F_{2}$ lot produced (1910) a sick lot of 3 white cocoons and 1 yellow cocoon. Mating (1908) another yellow and white pair of the same $F_{1}$ lot produced (1909) 10 white cocoons and 10 yellow cocoons. Mating (1909) two of these white cocooners produced (1910) 93 white cocoons and 25 yellow cocoons. Mating (1909) two of the $\mathrm{F}_{2}$ yellow cocooners produced (1910) 8 white cocoons and 35 yellow cocoons.

In 1907 a French Yellow female was mated with a Bagdad White male from $9: 40$ A.M. to 11:10 A.M. and then with a French Yellow male from 11:10 A.M. to 12.25 P.M. This mating produced 56 salmon ( $i$. e., pinkish yellow) cocoons and 34 salmon to golden yellow cocoons. (All of these in the general category yellow but varying in shade from pinkish yellow to deep old gold yellow). Mating (1908) two salmon cocooners produced (1910) 13 salmon cocoons and 3 white cocoons. Mating (1909) two of these $F_{2}$ salmon cocooners produced (1910) all salmon lot. Mating (1908) another salmon $\mathrm{F}_{1}$ pair produced (1909) 17 salmon cocoons and 8 white cocoons. Mating (1909) two of these $F_{2}$ salmon cocooners produced (1910) 10 salmon cocoons and 6 white cocoons.
So much for double matings between Bagdad Whites and French Yellows. Now for a series between Bagdad Whites and Istrian Yellows.

In 1907 an Istrian Yellow female was mated from 9 A.M. to $10: 30$ A.M. with an Istrian Yellow male and then from $10: 30$ until 12 with a Bagdad White male. This mating produced (1908) 55 yellow cocoons and one (straggler?) white cocoon. Mating (1908) two of the yellow cocooners produced (1909) 10 white cocoons and 23 yellow cocoons. Mating (1909) two of these yellow cocooners produced (1910) 2 white cocoons and 12 yellow cocoons. Mating (1908) another pair of yellow cocooners of the same $F_{1}$ lot produced (1909) 13 white cocoons and 28 yellow cocoons. Mating (1908) still another yellow pair from the same $\mathrm{F}_{1}$ lot produced (1909) 10 white cocoons and 24 yellow cocoons. Mating (1909) two of these white cocooners produced (1910) 4 white cocoons and no yellows. Mating (1909) two yellow cocooners of the same $F_{2}$ lot produced 16 white cocoons and no yellow cocoons.

In 1908 an Istrian Yellow female was mated with a Bagdad White male from 10:30 A.M. to 12 м. and then with an Istrian Yellow male from 12 to $1: 30$ P.M. This mating produced 30 yellow cocoons. Mating (1909) two of these yellows produced (1910) 16 yellow cocoons and 4 white cocoons. Mating (1909) another pair of the $\mathrm{F}_{1}$ yellows produced 19 yellow cocoons and 4 white cocoons.

In 1907 a Bagdad White female was mated with a Bagdad White male from $9: 45$ A.M. to 11 A.M. and then with an Istrian Yellow male from 11 A.M. to $12: 15$ P.M. This mating produced (1908) 15 white cocoons and 57 yellow cocoons. Mating (1908) two of these white cocooners together produced (1909) 11 white cocoons and no yellows. Two other pairs of white cocooners from the same $\mathrm{F}_{1}$ lot produced (1909) small all white lots. From each of these three all white $\mathrm{F}_{2}$ lots was mated (1909) one pair, and each mating produced (1910) a very small weak all white lot. Mating (1908) two yellow cocooners from the original $F_{1}$ generation lot produced (1909) 22 yellow cocoons and 6 white cocoons. Mating 
(1909) two of these white cocooners produced (1910) 35 white cocoons and no yellows. Mating (1909) two yellow cocooners produced 24 yellow cocoons and no whites. Mating (1908) another pair of $F_{1}$ yellow cocooners produced (1909) 9 white cocoons and 20 yellow cocoons. Mating (1909) two of these white cocooners together produced 25 white cocoons and no yellows. Mating (1909) two of the yellows together produced (1910) 12 white cocoons and 21 yellow cocoons. Mating (1908) another pair of yellow cocooners from the original $F_{1}$ lot produced (1909) 4 white cocoons and 17 yellow cocoons. Mating (1909) two of these yellow cocooners together produced 8 white cocoons and 20 yellow cocoons. Mating (1908) a yellow cocooner and white cocooner of the original $F_{1}$ lot produced (1909) 12 white cocoons and 12 yellow cocoons. Mating (1909) two of these white cocooners produced 19 white cocoons and no yellows. Mating (1909) two of the yellow cocooners produced (1910) 10 white cocoons and 17 yellow cocoons. Mating (1908) another yellow and white pair produced (1909) 16 white cocoons and 20 yellow cocoons. Mating (1909) two of these white cocooners produced (1910) 6 white cocoons and no yellows. Mating (1909) two yellows produced (1910) 8 white cocoons and 6 yellow cocoons. Mating (1909) a yellow and a white produced (1910) 4 white cocoons and 12 yellow cocoons. Mating (1908) another yellow and white pair produced (1909) 5 white cocoons and 31 yellow cocoons. Mating (1909) two of these whites produced 19 white cocoons and no yellows. Mating (1909) two of the yellows produced 9 yellow cocoons and no white cocoons.

In 1907 a Bagdad White female was mated with an Istrian Yellow male from $9: 45$ A.M to 11 A.M. and then with a Bagdad White male from 11 A.M to $12: 15$ P.M. This mating produced 41 white cocoons, many of them creamy white instead of the pure or faintly greenishwhite characteristic of the Bagdad white race. Mating (1908) two of these white cocooners produced (1909) 59 white cocoons. And mating (1909) two of these $F_{2}$ white cocooners produced (1910) a small all white lot. Similar $\mathrm{F}_{2}$ and $\mathrm{F}_{3}$ all white lots were obtained from another $F_{1}$ mating. Mating (1908) another pair of $\mathrm{F}_{1}$ white cocooners produced (1909) 46 white cocoons and 15 yellow cocoons. Mating (1909) two of these white cocooners produced (1910) a small all white lot. Mating (1909) two of these $F_{2}$ yellow cocooners produced (1910) an all yellow lot.

In 1907 a Bagdad White female was mated with an Istrian Yellow male from $9: 45$ A.M. to 11 A.M. and then with a Bagdad White male from 11 A.M. to $12: 15$ P.M. (This was an exact duplicate of the 1907 double mating just described.) This mating produced (1908) 48 white cocoons and 20 yellow cocoons. Mating (1908) two of these white cocooners produced (1909) a small all white lot, and a mating (1909) of two from this lot produced (1910) a smaller all white lot. Mating (1908) another white pair from the $F_{1}$ generation produced an all white lot, and a mating (1909) of two from this lot produced (1910) a small all white $\mathrm{F}_{3}$ lot. Mating (1908) two yellow cocooners of the $F_{1}$ lot produced 9 white cocoons and 12 yellow cocoons. Mating (1909) two of these $\mathrm{F}_{2}$ white cocooners produced a small all white lot, while mating (1909) two of the yellow cocooners produced (1910) a very small all yellow lot. Another mating (1908) of two yellow cocooners of the original $\mathrm{F}_{1}$ lot produced 26 yellow cocoons and one white cocoon and mating (1909) two of these $\mathrm{F}_{2}$ yellow cocooners produced (1910) 14 yellow cocoons and 2 white cocoons. Another mating (1908) of two yellow cocooners from the original $F_{1}$ lot produced 28 yellow cocoons and 12 white cocoons. Mating (1909) two of these $\mathrm{F}_{2}$ yellow cocooners produced 50 yellow cocoons and no white ones, while mating (1909) two of the white cocooners produced (1910) 15 white cocoons and 1 yellow cocoon (straggler?). Mating (1908) a yellow and a white from the original $F_{1}$ lot produced (1910) 40 white cocoons and 16 yellow cocoons. Mating (1909) two of these white cocooners produced (1910) 28 white cocoons and 29 yellow cocoons, while mating (1909) two of the $\mathrm{F}_{2}$ yellow cocooners produced (1910) 5 white cocoons 
and 34 yellow cocoons. Another mating (1908) of a yellow cocooner and a white cocooner from the original $\mathrm{F}_{1}$ lot produced (1909) 20 white cocoons and 19 yellow cocoons. Mating (1909) two of these white cocooners produced (1910) 70 white cocoons and no yellow ones, while mating (1909) two of these $\mathrm{F}_{2}$ yellow cocoons produced (1910) 6 white cocoons and 9 yellow cocoons.

In 1907 a Bagdad White female was mated with a male Istrian yellow from $9: 40$ A.M. to $10: 45$ A.M. and then with a male Bagdad White till death of the moths. This mating produced (1908) 29 yellow cocoons. Mating (1908) two of these yellow cocooners produced (1909) 25 yellow cocoons and 8 white cocoons. Mating (1909) two of these $\mathrm{F}_{2}$ whites produced (1910) a small all white lot. Mating (1908) another pair of the $\mathrm{F}_{1}$ yellow cocooners produced (1909) 6 white cocoons and 12 yellow cocoons. Mating (1909) two of these $\mathrm{F}_{2}$ white cocooners produced (1910) an all white lot. Mating (1909) two of the yellow cocoons produced 9 yellow cocoons and 6 white cocoons. Mating (1908) another pair of the $F_{1}$ yellow cocooners produced (1910) 30 yellow cocoons and 9 white cocoons. Mating (1909) two of these yellow cocooners produced (1910) an all yellow lot. Mating (1908) still another pair of the $\mathrm{F}_{1}$ yellow cocooners produced (1909) 19 yellow cocoons and 4 white cocoons. Mating (1909) two of these $\mathrm{F}_{2}$ yellow cocooners produced (1910) 29 yellow cocoons and 5 white cocoons.

These are the records. Their interpretation may be made by any one interested. In scrutinizing them for significance this should be remembered. In ordinary (single) matings of Bagdad White with Bagdad White only white cocoons are produced in $\mathrm{F}_{1}$ and all following generations. In mating French Yellow with French Yellow or Istrian Yellow with Istrian Yellow only yellow cocoons are produced in $\mathrm{F}_{1}$ and all following generations. In mating Bagdad White with Istrian YelJow usually all the cocoons of the $\mathrm{F}_{1}$ generation are yellow. Mating these together usually produces in $\mathrm{F}_{2}$ generations 3 yellow to 1 white, the Mendelian behavior. In mating Bagdad
White with French Yellow the dominance of yellow is not so steadfast. There is, as I have shown and particularly emphasized in $\mathrm{my}$ 1908 paper, more or less aberration from the Mendelian rules in this mating. And indeed, these aberrations are likely to occur in any other crossing of silkworm races. The usual inheritance behavior of silkworm cocoon characters is, however, Mendelian. The aberrations constitute what I have called "strain and individual idiosyncrasies." This simply means that I believe that there is more in the order of inheritance than is covered by Mendelism. The Mendelian elements in this order are becoming recognizable and familiar. The other elements are not yet so obvious to us.

In these double matings the aberrations are abundant and conspicuous. After a double mating the whites of the $F_{1}$ generation mated with other whites of the same generation do not always produce whites. They may produce both yellows and whites. Or this latent carrying of the yellow character by these presumably strictly recessive (white) carriers may not be manifest till an $\mathrm{F}_{3}$ generation. What does this mean?

In seeking an answer, the state of affairs as regards actual fertilization in these double mating cases must be kept in mind.

The female receives during an hour's or two hours' coupling a large number of fertilizing cells from a male of her own race (and hence her own cocoon characters). She then receives during another hour's or two hours' coupling a presumably equal number of germ cells from another male of different race (and different cocoon characters). These two lots of active spermatozoa are held in the spermatheca. Is one group above or in front of the other, so that when an egg arrives opposite the opening of the spermatheca it will necessarily be fertilized by one of this upper or front group (the group provided by the second male)? Or do the actively motile spermatozoa become thoroughly mingled in their fluid vehicle so that access to the egg will be according to the law of probabilities? More likely the latter alternative should prevail. 
When, however, a spermatozoan enters the egg through the micropyle this micropyle should, by analogy with the observed conditions in various other insect eggs, become closed, thus preventing poly-fertilization. If this is so then a double mating should after all result in but a single fertilization, and these fertilizations should be roughly divided between the two male types.

Thus in double mating a female Bagdad White with Bagdad White and Istrian Yellow males, the fertilizations should be, roughly, equally divided between pure race. Bagdad White and crossed Bagdad and Istrian Yellow. And in accordance with these fertilizations half of the $F_{1}$ generations thus produced should be white cocooning and half yellow cocooning (yellow being dominant in crossings with white). If an Istrian Yellow female is mated with both Istrian Yellow and Bagdad White males $F_{1}$ generations should always be composed of all yellow cocooning individuals. Or if in these double matings all of the fertilizations are effected by spermatozoa of one of the males only then the $F_{1}$ lots should be either all white cocooning or all yellow cocooning. $\mathrm{F}_{2}$ generations from these lots should follow the Mendelian order and break when the $F_{1}$ individuals are hybrids and not break when they are pure race progeny.

But the data given above do not reveal the expected behavior. They evidence a considerable perturbation in the order of inheritance. The $\mathrm{F}_{1}$ lots are not always all white or all yellow, or equally divided between white and yellow as they seemingly should be. Or if such all white or all yellow $\mathrm{F}_{1}$ lots are produced, they often throw both yellows and whites in $F_{2}$ lots when only yellows or only whites should have appeared. Or if they do produce all white or all yellow $\mathrm{F}_{2}$ lots intermating in these lots may produce both yellows and whites in $\mathrm{F}_{\mathbf{3}}$ lots. In a word the inheritance behavior is not that which it should be in animals usually following a Mendelian order, if the only influence at work on the egg is the nuclear content of a single pure race spermatozoan.
What, then, is causing this pertubation in the order of inheritance? Do the eggs in double-mated females receive more than one spermatozoan and are these spermatozoa often the representatives of both the races used in the double mating? Or can the egg be in any way influenced by the mere presence in the spermatheca of spermatozoa representing both of a pair of allelomorphic heritable characters? Can fluids carrying the spermatozoa have any influence during fertilization? Can the spermatozoa of one type influence those of the other type during their enforced companionship for several hours or days in the female spermatheca?

All that we think we know of the mechanism of fertilization and heredity makes us answer "No" to each of these questions. Then why should the order of inheritance in the silkworm moth be different in the generations after these double matings from the order in the generations following a single mating?

\section{Stanford University, Cal. \\ Vernon L. KellogG}

\section{SOCIETIES AND ACADEMIES}

THE NATIONAL ACADEMY OF SCIENCES

AT the stated meeting of the academy on April 18-20, the following papers were read:

"On the Motions of the Brighter Helium Stars,", W. W. Campbell.

" Report of Progress in Spectrographic Determinations of Stellar Motions,", W. W. Campbell.

"The Evolution of Periodic Solutions of the Problem of Three Bodies,' F. R. Moulton.

"'Mechanical Quadratures," G. F. Becker.

"Corollaries of the Theory of Isostasy," W. M. Davis.

"Experimental Investigation on Reflection of Light at Certain Metal-liquid Surfaces," Lynde P. Wheeler (introduced by C. H. Hastings).

"On the Origin of the Peaks of Maximum Pressure in the Midst of the Permanent Tropical Oceanic Highs," W. J. Humphreys (introduced by Cleveland Abbe).

"A Further Study of Columbic and Tantallic Oxides,", E. F. Smith.

"The Outlook of Petrology," J. P. Iddings.

"The Orogenic Development of the Northern Sierra Nevada," Waldemar Lindgren. 